\title{
Studies on the role of ACTH in the regulation of adrenal responsiveness and the timing of parturition in the ovine fetus
}

\author{
K R Poore, I R Young, B J Canny and G D Thorburn
}

Department of Physiology, Monash University, Clayton, Victoria 3168, Australia

(Requests for offprints should be addressed to I R Young)

\begin{abstract}
A dramatic late-gestation increase in fetal plasma cortisol concentrations is critical for the timing of parturition in the sheep. This increase appears to depend upon an intact hypothalamo-pituitary unit and is characterised by increasing responsiveness of the fetal adrenal gland to ACTH. ACTH has been postulated as the critical determinant of the late-gestation cortisol increase; however, recent evidence has suggested that other factors, including the ACTH precursor, pro-opiomelanocortin, may also be involved. To further define the role of ACTH in determining the timing of parturition and the responsiveness of the fetal adrenal gland, intact (INT/ACTH) and hypophysectomised (HX/ACTH) fetuses received a continuous infusion of $\mathrm{ACTH}_{(1-24)}$ from the time of surgery $(\sim 115$ days gestational age $(\mathrm{GA}))$ at a rate we have previously shown to generate normal fetal cortisol concentrations and term parturition in HX fetuses. A third group of saline-infused intact fetuses (INT/SAL) served as the control group. Adrenal responsiveness was assessed by cortisol responses to $\mathrm{ACTH}_{(1-24)}$ challenges at 120, 130 and 140 days GA.

There were no differences between the three groups of fetuses in the timing of parturition, the late-gestation increase in cortisol concentrations or the size of the adrenal cortex. In both INT/SAL and INT/ACTH fetuses, there were significant increases in basal immunoreactive-ACTH
\end{abstract}

concentrations with advancing GA, although no such increase was observed in HX/ACTH fetuses. The proportion of total ACTH immunoreactivity present in low molecular weight (LMW) forms in INT/ACTH fetuses was greater than that in INT/SAL fetuses, while the level of LMW ACTH in HX/ACTH fetuses was intermediate. Both $\mathrm{ACTH}_{(1-24)}$-infused groups of fetuses had dramatically enhanced adrenal responsiveness to $\mathrm{ACTH}_{(1-24)}$ at all GAs tested when compared with INT/SAL fetuses and there was a correlation (in rank order) between the proportion of LMW ACTH immunoreactivity and adrenal responsiveness.

From these observations it appears that there is a separate regulation of adrenal responsiveness from basal cortisol concentrations and that an increase in basal cortisol concentrations can occur in the absence of an increase in basal ACTH concentrations. Furthermore, an increase in adrenal responsiveness does not appear to predict the timing of parturition nor basal cortisol concentrations. Taken together with previous studies it appears that ACTH plays an essential role in maintaining the growth of the fetal adrenal and enhancing its responsiveness, but a late-gestation increase in ACTH concentrations is not required to regulate basal cortisol concentrations or the timing of parturition.

Journal of Endocrinology (1998) 158, 161-171

\section{Introduction}

It has long been recognised that parturition in the sheep is dependent upon activation of the fetal hypothalamopituitary-adrenal (HPA) axis (Thorburn \& Liggins 1994). In the last 2 weeks of gestation there is a characteristic increase in plasma cortisol concentrations which is widely believed to reflect an increase in fetal adrenocorticotrophin (ACTH) concentrations (Challis \& Brooks 1989, Thorburn \& Liggins 1994). The role of ACTH in the timing of parturition was implied by early studies which showed that fetal hypophysectomy (HX) causes adrenal atrophy and prevents delivery (Liggins et al. 1967), while ACTH infusion at pharmacological doses induces an increase in plasma cortisol levels and premature parturition (Liggins 1968). Two more recent lines of evidence from our laboratory have, however, led us to question the obligatory role of an increase in fetal ACTH secretion in the initiation of parturition. First, we have demonstrated that labour is not established in fetuses that have undergone hypothalamopituitary disconnection, despite normal or elevated ACTH concentrations and an apparent increase in ACTH concentrations in late gestation (Antolovich et al. 1991, Ozolins et al. 1992, Deayton et al. 1994). Secondly, we have recently shown that $\mathrm{HX}$ fetuses continuously infused with a steady-state maintenance dose of $\mathrm{ACTH}_{(1-24)}$ are delivered at normal term with a normal pre-parturient increase in cortisol concentrations (Jacobs et al. 1994). 
The changing responsiveness of the ovine fetal adrenal gland to ACTH is also believed to contribute to rising cortisol concentrations prior to labour. In vitro and in vivo studies have shown that the fetal adrenal gland becomes increasingly responsive to ACTH stimulation in the last few weeks of gestation (Wintour et al. 1975, Jones et al. 1977, Glickman \& Challis 1980, Rose et al. 1982). Jones \& Roebuck (1980) suggested that the ability of the ovine fetal adrenal gland to respond to circulating ACTH in vivo was suppressed by some factor(s), since they found a discrepancy between adrenal cortisol responses to ACTH in vitro and in vivo. In support of this proposal, adrenal cells from 120-day-old fetuses, maintained in an ACTH-free medium, exhibit enhanced responsiveness as the time in culture increases, suggesting the existence of an inhibitor of ACTH action in vivo (Durand et al. 1982). It has been suggested that these putative inhibitory factors are the high molecular weight (HMW) precursor peptides of ACTH itself, since these peptides have been shown to inhibit ACTH-induced cortisol secretion from adrenal cells in vitro (Roebuck et al. 1980, Jones et al. 1992, Schwartz et al. 1995). As the HMW ACTH-containing peptides are present in fetal plasma in high concentrations (Saphier et al. 1993, Carr et al. 1995), recent attention has therefore focused on the ratio of low molecular weight (LMW) to HMW ACTH, since it has been proposed that the balance between the stimulatory action of ACTH and the proposed inhibitory action of the HMW ACTH precursor peptides is involved in the regulation of fetal adrenal responsiveness and basal cortisol secretion (Roebuck et al. 1980, Durand et al. 1982, Schwartz et al. 1995). It has been speculated that the successful establishment of labour in HX fetuses receiving a constant maintenance infusion of $\mathrm{ACTH}_{(1-24)}$ reflects an enhanced responsiveness of the adrenal glands of these fetuses to ACTH (Jacobs et al. 1994).

The present study was undertaken to determine the effect of a constant maintenance $\mathrm{ACTH}_{(1-24)}$ infusion, in fetuses with intact and disrupted hypothalamo-pituitary function, on the timing of labour, basal ACTH and cortisol concentrations and the molecular weight profile of ACTH-containing peptides. In addition, we aimed to formally test the responsiveness of the fetal adrenal gland to an $\mathrm{ACTH}_{(1-24)}$ challenge in $\mathrm{ACTH}_{(1-24)}$-infused intact and HX fetuses.

\section{Materials and Methods}

\section{Animals}

All procedures involving the use of animals were approved by the Standing Committee on Ethics in Animal Experimentation of Monash University.

Border Leicester-Merino cross-bred ewes of known gestational age (GA) were used in this study. The ewes were housed in individual metabolism cages and fed between $0900 \mathrm{~h}$ and $1200 \mathrm{~h}$ daily. Water was freely available. Surgery was performed on ewes between 107 days and $119(115 \pm 1)$ days GA using aseptic techniques. Fetuses underwent HX, as previously described (Mesiano et al. 1987), or were left intact (INT). Vascular catheters were implanted into a jugular vein and common carotid artery of all fetuses. In most ewes, a maternal jugular vein was catheterised and electromyographic (EMG) electrodes were sutured to the myometrium to record uterine EMG activity for the detection of labour. Fetal well-being was monitored by measuring fetal arterial blood gases using an ABL30 blood gas analyser and OSM2 hemoximeter (Radiometer, Copenhagen, Denmark). At the end of each experiment, ewes and fetuses were killed by barbiturate overdose (Lethabarb, Arnolds of Reading Pty Ltd, Boronia, Victoria, Australia) administered via the maternal jugular vein catheter.

\section{Experiment 1: gestational outcome, adrenal morphometry and} basal hormone concentrations

A total of 28 fetuses was used in this experiment. Eleven fetuses were left intact and received a continuous saline infusion $(0.9 \% \mathrm{NaCl}$ (Baxter Healthcare Pty Ltd, Old Toongabbie, NSW, Australia) containing $50000 \mathrm{IU}$ heparin/l (Fisons Pty Ltd, Sydney, Australia); $1 \mathrm{ml} / \mathrm{h}$ ); eight fetuses were left intact and received a continuous maintenance infusion of $\mathrm{ACTH}_{(1-24)}$ (Synacthen, Ciba Geigy Australia Ltd, Pendle Hill, NSW, Australia; $43 \mathrm{ng} / \mathrm{h}$ per kg estimated fetal body weight) in heparinised saline; and a further nine fetuses underwent HX and also received a continuous maintenance infusion of $\mathrm{ACTH}_{(1-24)}$ in heparinised saline. One of the fetuses in the HX/ACTH group was a twin; both fetuses underwent $\mathrm{HX}$, but only one received the $\mathrm{ACTH}_{(1-24)}$ infusion. Two ewes in the intact saline treated (INT/SAL) group carried twins, and in one of these ewes both twins were studied. All of the INT/ACTH fetuses were singletons. All infusions commenced immediately following surgery. The $\mathrm{ACTH}_{(1-24)}$ infusion rate is exactly the same as that used previously by Jacobs et al. (1994) but in the previous publication the dose was misreported as $100 \mathrm{ng} / \mathrm{h}$ per $\mathrm{kg}$. The infusions continued until the end of the study and the dose was increased every 5 days in accordance with the estimated increase in fetal body weight (Cloete 1939).

The study continued until either the uterine EMG activity indicated the onset of labour or the length of gestation reached 161 days. At post mortem examination, fetal body, adrenal gland and pituitary weights (where present) were recorded. Adrenal glands were fixed in 10\% formaldehyde for adrenal morphometry (see below). At post mortem of HX fetuses, the pituitary fossa was examined to ensure completeness of HX. Of at least 50 fetuses that have undergone HX in our laboratory, none has delivered without exogenous stimulation of the adrenal 
gland (authors' unpublished observations and Mesiano et al. 1987 and Jacobs et al. 1994).

Routine blood sampling Starting 3 days after surgery, fetal arterial blood samples were collected every 2 or 3 days for the measurement of basal immunoreactive (ir)-ACTH and cortisol concentrations. Blood samples $(3 \mathrm{ml})$ were collected into chilled tubes containing EDTA $(5.6 \mathrm{mg}$ in $50 \mu \mathrm{l} ; 50 \mu \mathrm{l} / 3 \mathrm{ml}$ blood; BDH Chemicals Australia Pty Ltd, Kilsyth, Victoria, Australia). Once every 5 days, blood samples $(4 \mathrm{ml})$ were taken into tubes containing an ACTH degradation inhibitor mix $(80 \mu \mathrm{l}$; contains aprotinin (1000 kallikrein inhibitor units/ml), N-ethyl-maleimide (25 mg/ml, Sigma Chemical Company, St Louis, MO, USA) and EDTA (18.6 mg/ml, BDH Chemicals) in $0.9 \%$ $\mathrm{NaCl}$ ) for chromatographic analysis of ACTH-containing peptides. All blood samples were centrifuged for $5 \mathrm{~min}$ at 3000 r.p.m. at $4{ }^{\circ} \mathrm{C}$ and the plasma aliquots were stored immediately at $-20^{\circ} \mathrm{C}$ until assayed. ACTH degradation inhibitor mix was added to plasma collected for analysis of ir-ACTH concentration $(20 \mu \mathrm{l} / \mathrm{ml}$ plasma). Plasma for ACTH chromatography was acidified with $1.6 \%$ glycine in $0 \cdot 1 \mathrm{M} \mathrm{HCl}(150 \mu \mathrm{l} / \mathrm{ml}$ plasma $)$.

Experiment 2: the effect of $A C T H_{(1-24)}$ infusion on fetal adrenal responsiveness

A total of 14 fetuses from Experiment 1 was used in these experiments (INT/SAL, $n=4$; INT/ACTH, $n=4$; HX/ ACTH, $n=6)$. Commencing at each of 120,130 and 140 days GA, ACTH $\mathrm{A}_{(1-24)}$ was administered to each fetus by rapid intravenous injection at approximately the same time of day (approximately $1500 \mathrm{~h}$ ). On the first experimental day, $1.0 \mu \mathrm{g} / \mathrm{kg} \mathrm{ACTH} \mathrm{Al-24}_{(1)}$ (in $5 \mathrm{ml}$ heparinised saline) was administered, and 24 or $48 \mathrm{~h}$ later, $5.0 \mu \mathrm{g} / \mathrm{kg}$ $\mathrm{ACTH}_{(1-24)}$ was given. Fetal arterial blood samples $(3 \mathrm{ml})$ were collected at $30 \mathrm{~min}, 15 \mathrm{~min}$ and immediately prior to $\mathrm{ACTH}_{(1-24)}$ injection, and at 10, 30, 60, 90 and $120 \mathrm{~min}$ following the injection for analysis of cortisol concentrations. Samples were collected and stored as for routine blood samples (see above). Blood cells were returned to the fetus at the end of the experiment in an equal volume of Hartmann's Solution (Baxter Healthcare).

Adrenal responsiveness for each $\mathrm{ACTH}_{(1-24)}$ challenge experiment was measured by integrating the plasma cortisol response to $\mathrm{ACTH}_{(1-24)}(10-120 \mathrm{~min})$ above the mean pre-injection ( -30 to $0 \mathrm{~min}$ ) cortisol concentrations. The increase in cortisol concentration was also calculated as an index of adrenal responsiveness and was given by the peak cortisol concentrations achieved following $\mathrm{ACTH}_{(1-24)}$ administration minus the mean preinjection concentrations. It is important to note that this experimental protocol assesses adrenal responsiveness (i.e. the ability of the adrenal gland to produce cortisol in response to given doses of $\left.\mathrm{ACTH}_{(1-24)}\right)$ rather than the sensitivity of the adrenal to $\mathrm{ACTH}_{(1-24)}$ (i.e. the $\mathrm{ED}_{50}$ of
$\mathrm{ACTH}_{(1-24)}$ in inducing adrenal cortisol production). This approach was taken as it was not feasible to determine full dose-response relationships at each GA and because responsiveness is considered to be the important parameter changed in the pre-partum adrenal gland (Durand et al. 1985).

Experiment 3: the effect of interrupting the $A C T H_{(1-24)}$ infusion

To assess the contribution of the $\mathrm{ACTH}_{(1-24)}$ infusion to basal ir-ACTH and cortisol concentrations in INT/ ACTH $(n=4)$ and HX/ACTH $(n=5)$ fetuses, the $\mathrm{ACTH}_{(1-24)}$ infusion was turned off for a period of $4 \mathrm{~h}$ between 132 days and $138(136 \pm 1)$ days GA. Three HX/ACTH and two INT/ACTH fetuses had been used in Experiment 1, but not Experiment 2, while an additional four fetuses were used solely for this experiment. Fetal arterial blood samples $(3 \mathrm{ml})$ were collected, as for routine blood samples (see above), for ir-ACTH and cortisol radioimmunoassay (RIA; see below), at -30 , $-15,0,15,30,45,60,120,180,240,255,270,285$, 300,330 and $360 \mathrm{~min}$ relative to the time when the $\mathrm{ACTH}_{(1-24)}$ infusion was turned off. Blood cells were returned to the fetus at regular interval throughout the experiment (see above). Ir-ACTH concentrations were measured using the ICN RIA (see below).

\section{General methods}

Adrenal morphometry Following fixation, the adrenal glands from a subset of fetuses in Experiment 1 were embedded in paraffin wax and sectioned $(10 \mu \mathrm{m})$, transverse to the long axis, in the mid-glandular region. Sections were stained with haematoxylin and eosin for examination by light microscopy. A magnetic optical plate (Cadmate, CM-1212A, SDR Clinical Technology, Middle Cove, NSW, Australia) interfaced with a personal computer was used to measure the areas represented by the adrenal cortex and the whole adrenal. Results from six sections from each animal were averaged.

Chromatographic analysis of ACTH-containing peptides To separate ir-ACTH species of differing MW, plasma samples $(1 \mathrm{ml})$ collected for chromatographic analysis (Experiment 1) were individually thawed and loaded onto a Sephadex G50 Fine gel filtration chromatography column (Pharmacia LKB, Uppsala, Sweden), as previously described (Hollingworth et al. 1995). The sample was eluted with $1 \%$ formic acid containing $1 \mathrm{~g} / 1$ Polypep (Sigma) at a rate of $10 \mathrm{ml} / \mathrm{h}$. Fractions $(2 \mathrm{ml})$ were collected, lyophilised in a rotary evaporator and stored at $-20{ }^{\circ} \mathrm{C}$. Lyophilised fractions were reconstituted in assay buffer $(0.3 \mathrm{ml})$ and left at room temperature for approximately $2 \mathrm{~h}$ prior to assay for ir-ACTH concentration using the Monash RIA (see below). HMW and 
Table 1 Gestation lengths, fetal body weights, combined fetal adrenal weights, adrenal/body weight ratios and the ratio of the adrenal cortex to the total adrenal area at term in INT/SAL $(n=7$, except for adrenal area measurements where $n=3)$, INT/ACTH $(n=6$, except for adrenal area measurements where $n=4)$ and HX/ACTH $(n=7$, except for adrenal area measurements where $n=4$ ) fetuses (only those from ewes that went into labour). Data are means \pm S.E.M.

\begin{tabular}{|c|c|c|c|}
\hline & INT/SAL & INT/ACTH & HX/ACTH \\
\hline Gestation length (days) & $149 \pm 1$ & $145 \pm 1$ & $148 \pm 2$ \\
\hline Body weight $(\mathrm{kg})$ & $4 \cdot 8 \pm 0 \cdot 3^{a}$ & $3 \cdot 8 \pm 0 \cdot 4^{b}$ & $3 \cdot 5 \pm 0 \cdot 2^{b}$ \\
\hline Adrenal weight (g) & $0.65 \pm 0.06$ & $0.66 \pm 0.08$ & $0 \cdot 51 \pm 0 \cdot 03$ \\
\hline Adrenal/body weight ratio (g/kg) & $0 \cdot 14 \pm 0 \cdot 01$ & $0 \cdot 17 \pm 0 \cdot 02$ & $0 \cdot 15 \pm 0 \cdot 01$ \\
\hline Cortical/total adrenal area & $0 \cdot 68 \pm 0 \cdot 02$ & $0 \cdot 67 \pm 0 \cdot 03$ & $0 \cdot 65 \pm 0 \cdot 03$ \\
\hline
\end{tabular}

Different letters indicate values that are significantly different from each other as indicated by least squares difference (LSD) test.

LMW ir-ACTH concentrations in each sample were calculated as the sum of ir-ACTH concentrations in fractions 8-11 and the sum of ir-ACTH concentrations in fractions 14-16 respectively. LMW ir-ACTH concentrations were expressed as a percentage of total ir-ACTH (i.e. the sum of LMW and HMW ir-ACTH concentrations).

Radioimmunoassays Fetal plasma cortisol concentrations were measured by RIA after extraction with dichloromethane, as previously described (Bocking et al. 1986). The intra- and inter-assay coefficients of variation were 5 and $18 \%$ respectively, at a value of $12.8 \pm 0.5 \mathrm{ng} /$ $\mathrm{ml}$. The sensitivity of the assay was $1 \cdot 2 \pm 0 \cdot 3 \mathrm{ng} / \mathrm{ml}$. The average recovery for the cortisol extraction procedure was $95 \pm 1 \%$. Ir-ACTH concentrations in chromatographic fractions and unextracted fetal plasma were measured in an RIA established at Monash University for these purposes (Hollingworth et al. 1995). The intra- and inter-assay coefficients of variation for the Monash RIA were 7 and $20 \%$ respectively, at a value of $1518 \cdot 8 \pm 65 \cdot 5 \mathrm{pg} / \mathrm{ml}$. The sensitivity of the assay was $16 \cdot 8 \pm 4 \cdot 1 \mathrm{pg} / \mathrm{ml}$. Ir-ACTH concentrations in selected samples of unextracted fetal plasma were also measured using a commercially available RIA kit (ICN Biomedicals Australasia Pty Ltd, Seven Hills, NSW, Australia). The intra- and inter-assay coefficients of variation for the ICN RIA were 10 and $7 \%$ respectively, at a value of $147 \cdot 0 \pm 4 \cdot 4 \mathrm{pg} / \mathrm{ml}$. The sensitivity of this assay was $8.5 \pm 1 \cdot 0 \mathrm{pg} / \mathrm{ml}$. Both of these ACTH antisera cross react with peptides containing the $\mathrm{ACTH}_{(1-24)}$ sequence, including the HMW precursor peptides of ACTH. There was a significant $(P<0 \cdot 001)$ correlation between ir-ACTH concentrations in the same plasma samples when measured using each of these ACTH RIAs. The regression equation in the analysis was: $y=1 \cdot 3 x+123$ where $y=[$ ir $-A C T H](p g / m l)$ using the Monash RIA and $\mathrm{x}=[\mathrm{ir}-\mathrm{ACTH}](\mathrm{pg} / \mathrm{ml})$ using the ICN RIA $(n=104, r=0 \cdot 71)$.

Statistics All results are expressed as means \pm standard error of the mean (S.E.M.). Data were first tested for homogeneity of variance using Bartlett-Box F and Cochran's C tests. Data found heterogeneous were rendered homogeneous by square root or logarithmic transformation. The effects of experimental group, GA, time and individual animals were tested using multifactorial ANOVA for repeated measures. Where appropriate, least significant difference (LSD) tests were applied to identify differences between means. Statistical significance is reported at the $0 \cdot 05$ level.

\section{Results}

Experiment 1: gestational outcome and basal hormone concentrations

Fetal outcome One ewe in the HX/ACTH group did not show any signs of labour by 161 days GA, at which time she and the fetus were killed. The fetus of another ewe in this group died at 151 days GA (due to an intrauterine infection) before any signs of labour. Results from these fetuses were excluded from analyses of basal hormone concentrations since the day of labour onset could not be determined and hence results could not be adjusted to the day of labour. All other ewes had apparently normal labour.

The mean gestation lengths, fetal body weights, combined fetal adrenal weights, adrenal/body weight ratios and the ratio of the adrenal cortex to the total adrenal area at term for each group of fetuses (from ewes that went into labour) are shown in Table 1. There were no significant differences in gestation lengths between the three groups of fetuses. Body weights at term in INT/ACTH and $\mathrm{HX} / \mathrm{ACTH}$ fetuses were significantly reduced compared with those in INT/SAL fetuses. There were no significant differences in adrenal weights between the three groups of fetuses and the mean adrenal/body weight ratios at term were not significantly different between the three groups. The adrenal/body weight ratio of the HX/ACTH fetus that failed to deliver by 161 days GA was $0 \cdot 08$. This is substantially less than those of HX/ACTH fetuses that delivered (mean $0 \cdot 15,95 \%$ confidence limits $0 \cdot 13-0 \cdot 17$, 
see Table 1), and is similar to that of untreated HX fetuses killed at 147 days (Mesiano et al. 1987). There were no significant differences in the ratios of the areas of the adrenal cortex to the total adrenal area at term between the three groups of fetuses.

Basal cortisol concentrations There were no significant differences in basal cortisol concentrations between the three groups of fetuses over the last 28 days of gestation (Fig. 1A). In all groups of fetuses, there was a significant increase in cortisol concentrations with advancing GA which first reached statistical significance, as determined by LSD test, 14 days before labour (when compared with the first three GA points). In the HX/ACTH fetus that did not deliver, basal cortisol concentrations rose to $80 \mathrm{ng} / \mathrm{ml}$ at 155 days GA, but then fell dramatically to $10 \mathrm{ng} / \mathrm{ml}$ by 161 days GA, while in the HX/ACTH fetus that suffered an intrauterine death at 151 days, fetal cortisol concentrations were increasing until the time of death.

\section{Basal ir-ACTH concentrations}

Monash RIA There were no significant differences in basal plasma ir-ACTH concentrations as measured by the Monash RIA between the three groups of fetuses (Fig. 1B). In both INT/SAL and INT/ACTH fetuses, there were significant increases in ir-ACTH concentrations in the last 4 days of gestation (when compared with the first three time points, as determined by LSD test). In HX/ACTH fetuses, however, at no time point could the ir-ACTH concentrations be identified as significantly different from control values (the first three time points) by LSD test, indicating no significant increase in ir-ACTH concentrations prior to labour. The ir-ACTH concentrations in the HX/ACTH fetuses that did not deliver, or suffered an intrauterine death, were not substantially different from other fetuses in this group.

ICN RIA As the results obtained using the Monash RIA suggested that increases in ir-ACTH concentrations did not occur in the HX/ACTH fetuses, we wished to confirm this observation by using a completely independent assay. Accordingly, ir-ACTH concentrations were assessed in selected plasma samples using a commercially available ACTH RIA (ICN RIA kit). Since not all samples were available for assay, the study period was divided into six GA blocks, each 5 days in length, and one sample per block (the latest in GA possible) per animal was assayed (Table 2). For comparison, results obtained using the Monash RIA for all samples within each of these GA blocks (two or three samples) were averaged and are also presented in Table 2. There were no significant differences in basal plasma ir-ACTH concentrations as measured using the ICN RIA between the three groups of fetuses. In both INT/SAL and INT/ACTH fetuses, there were significant increases in basal ir-ACTH concen- trations (when compared with the first two GA blocks, as determined by LSD test) in the last 9 days of gestation. In contrast, there were no significant changes in basal irACTH concentrations with advancing GA in HX/ACTH fetuses. Analysis of ir-ACTH concentrations as measured using the Monash RIA and presented in this format revealed the same pattern of results; i.e. no effect of experimental group and significant increases in ir-ACTH concentrations with advancing GA in INT/SAL and INT/ACTH fetuses but not HX/ACTH fetuses.

Percentage of LMW ir-ACTH in plasma samples Since samples for the assessment of LMW ir-ACTH were taken every 5 days, data obtained for the percentage of LMW ir-ACTH as a proportion of total ir-ACTH are presented in six GA blocks, each 5 days in length (as described above for ir-ACTH concentrations). There was a significant effect of treatment group on the percentage of LMW ir-ACTH (Fig. 2). The percentage of LMW ir-ACTH in plasma from INT/ACTH fetuses was significantly greater than that in INT/SAL fetuses, while the percentage of LMW ir-ACTH in plasma from HX/ ACTH fetuses was intermediate between, and not different from, that of INT/SAL and INT/ACTH fetuses. In plasma from INT/SAL fetuses, there was a significant increase in the percentage of LMW ir-ACTH in the last 4 days of gestation (when compared with the first two GA blocks). There were no significant changes in the percentage of LMW ir-ACTH with advancing GA in plasma from INT/ACTH or HX/ACTH fetuses.

\section{Experiment 2: the effect of $A C T H_{(1-24)}$ infusion on fetal adrenal responsiveness}

Adrenal responsiveness was assessed by measuring both the area under the cortisol response curve (Fig. 3) and the increase in cortisol concentrations achieved (Table 3) following the administration of $1.0 \mu \mathrm{g} / \mathrm{kg}$ and $5.0 \mu \mathrm{g} / \mathrm{kg}$ ACTH $_{(1-24)}$ to INT/SAL, INT/ACTH and HX/ACTH fetuses at 120, 130 and 140 days GA. Identical statistical conclusions were drawn from the assessment of both indices of adrenal responsiveness, and for clarity in the following description the term adrenal responsiveness encompasses both area under the cortisol response curve and the increase in cortisol concentrations achieved. It is of note that the HX/ACTH fetus that did not deliver, and that which suffered an intrauterine death, responded to the exogenous $\mathrm{ACTH}_{(1-24)}$ so their results have been included in the group data for the HX/ACTH fetuses. There was a significant effect of treatment on adrenal responsiveness. Adrenal responsiveness was significantly greater in both groups receiving $\mathrm{ACTH}_{(1-24)}$ infusion (i.e. INT/ACTH and HX/ACTH fetuses) than in INT/SAL fetuses. There was no significant difference in adrenal responsiveness between INT/ACTH and HX/ACTH fetuses. In all groups of fetuses, adrenal responsiveness significantly 

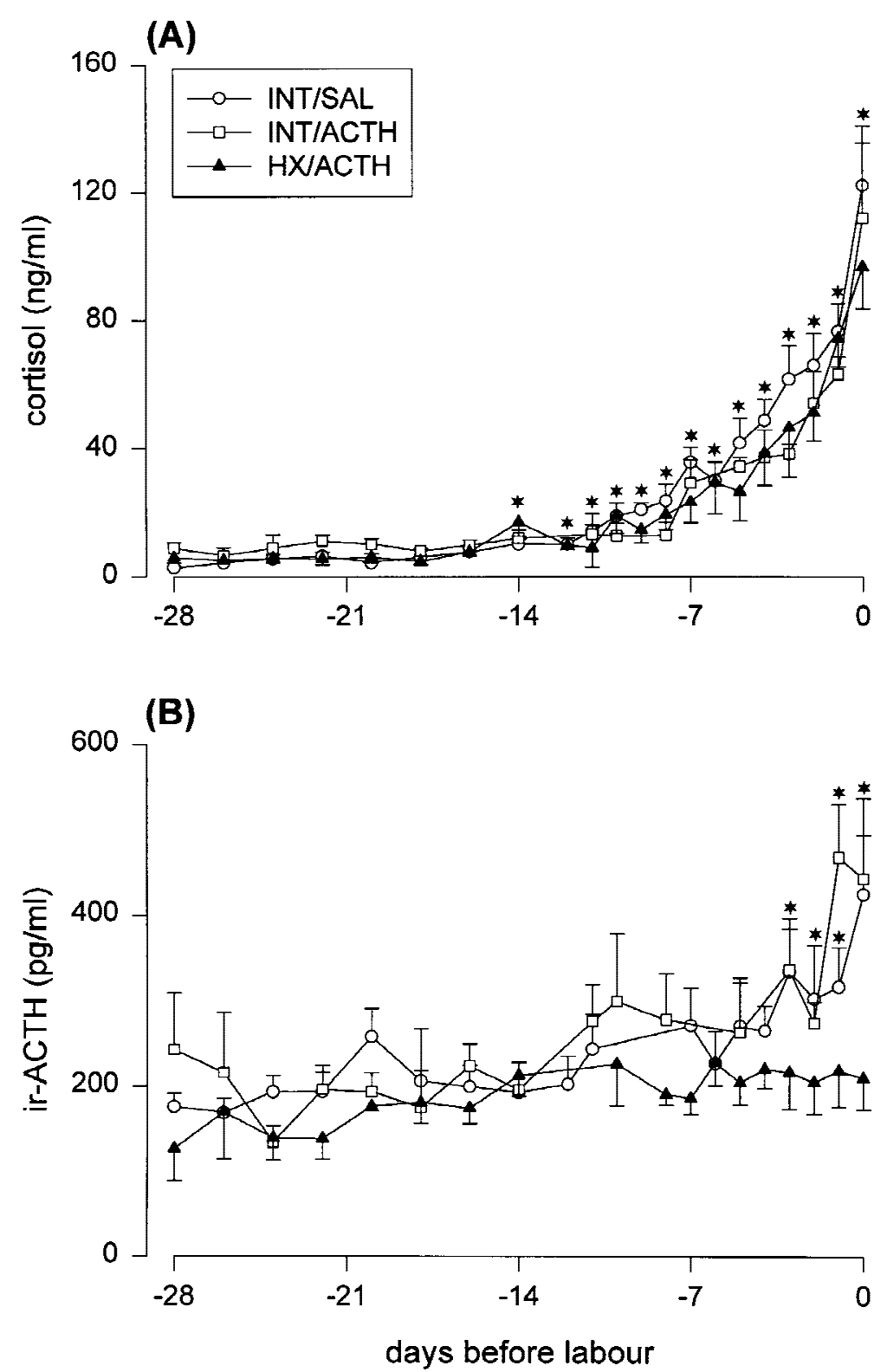

Figure 1 Plasma cortisol (A) and ir-ACTH (B) concentrations (as measured by the Monash RIA) during the study period in INT/SAL $(n=7)$, INT/ACTH $(n=6)$ and $\mathrm{HX} / \mathrm{ACTH}(n=7)$ fetuses (only those from ewes that went into labour). There were no significant differences in basal cortisol concentrations between the three groups of fetuses. In all groups of fetuses, there was a significant increase in cortisol concentrations with advancing GA. There were no significant differences in basal ir-ACTH concentrations between the three groups of fetuses. There were significant increases in ir-ACTH concentrations in INT/SAL and INT/ACTH fetuses but not $\mathrm{HX} / \mathrm{ACTH}$ fetuses. Asterisks indicate values, for each hormone, that are significantly different (from the first three GA points) as indicated by LSD test.

increased with advancing GA, and there was a significant effect of $\mathrm{ACTH}_{(1-24)}$ challenge dose on adrenal responsiveness. There were no significant differences in basal cortisol concentrations prior to each $\mathrm{ACTH}_{(1-24)}$ challenge experiment (each $\mathrm{ACTH}_{(1-24)}$ dose) at any GA, in any group of fetuses (data not shown).

The mean area under the cortisol response curve (for all GAs) in INT/SAL fetuses was $481 \pm 117$ ng.min per ml, 
Table 2 Plasma ir-ACTH concentrations ( $\mathrm{pg} / \mathrm{ml}$ ) from 29-25 days before labour (DBL) to $5-0$ days before labour in INT/SAL ( $n=7$ ), INT/ACTH $(n=6)$ and HX/ACTH $(n=7)$ fetuses (only those from ewes that went into labour) as measured by the ICN RIA and the Monash RIA. Data are means \pm S.E.M.

\section{ICN RIA}

\begin{tabular}{|c|c|c|c|}
\hline \multirow[b]{2}{*}{ DBL } & INT/SAL & INT/ACTH & HX/ACTH \\
\hline & & & \\
\hline $29-25$ & $33 \cdot 8 \pm 5 \cdot 7$ & $40 \cdot 2 \pm 1 \cdot 5$ & $44 \cdot 5 \pm 30 \cdot 3$ \\
\hline $24-20$ & $56 \cdot 6 \pm 6 \cdot 9$ & $41 \cdot 1 \pm 5 \cdot 4$ & $65 \cdot 6 \pm 18 \cdot 9$ \\
\hline $19-15$ & $73 \cdot 1 \pm 23 \cdot 3$ & $51 \cdot 0 \pm 4 \cdot 9$ & $44 \cdot 5 \pm 7 \cdot 9$ \\
\hline $14-10$ & $58 \cdot 2 \pm 6 \cdot 3$ & $60 \cdot 0 \pm 9 \cdot 2$ & $56 \cdot 8 \pm 18 \cdot 3$ \\
\hline $9-5$ & $107 \cdot 9 \pm 14 \cdot 8^{*}$ & $79 \cdot 2 \pm 13 \cdot 0^{*}$ & $70 \cdot 5 \pm 16 \cdot 7$ \\
\hline $4-0$ & $226 \cdot 0 \pm 77 \cdot 0^{*}$ & $155 \cdot 4 \pm 38 \cdot 5^{*}$ & $114 \cdot 3 \pm 48 \cdot 1$ \\
\hline
\end{tabular}

Monash RIA

\begin{tabular}{|c|c|c|}
\hline INT/SAL & INT/ACTH & HX/ACTH \\
\hline $166 \cdot 2 \pm 11 \cdot 3$ & $197 \cdot 3 \pm 51 \cdot 1$ & $173 \cdot 4 \pm 53 \cdot 6$ \\
\hline $196 \cdot 8 \pm 26 \cdot 7$ & $177 \cdot 0 \pm 18 \cdot 1$ & $157 \cdot 2 \pm 19 \cdot 5$ \\
\hline $203 \cdot 1 \pm 24 \cdot 6$ & $195 \cdot 0 \pm 25 \cdot 5$ & $117 \cdot 2 \pm 17 \cdot 7$ \\
\hline $204 \cdot 2 \pm 30 \cdot 0$ & $248 \cdot 1 \pm 20 \cdot 8$ & $227 \cdot 5 \pm 24 \cdot 9$ \\
\hline $267 \cdot 2 \pm 54 \cdot 1^{*}$ & $275 \cdot 8 \pm 46 \cdot 6$ & $201 \cdot 7 \pm 16 \cdot 8$ \\
\hline $335 \cdot 1 \pm 37 \cdot 2^{*}$ & $401 \cdot 6 \pm 42 \cdot 6^{*}$ & $196 \cdot 6 \pm 29 \cdot 2$ \\
\hline
\end{tabular}

Asterisks indicate values, for that variable, that are significantly different from others (when compared to the first two GA blocks ) as indicated by LSD test.

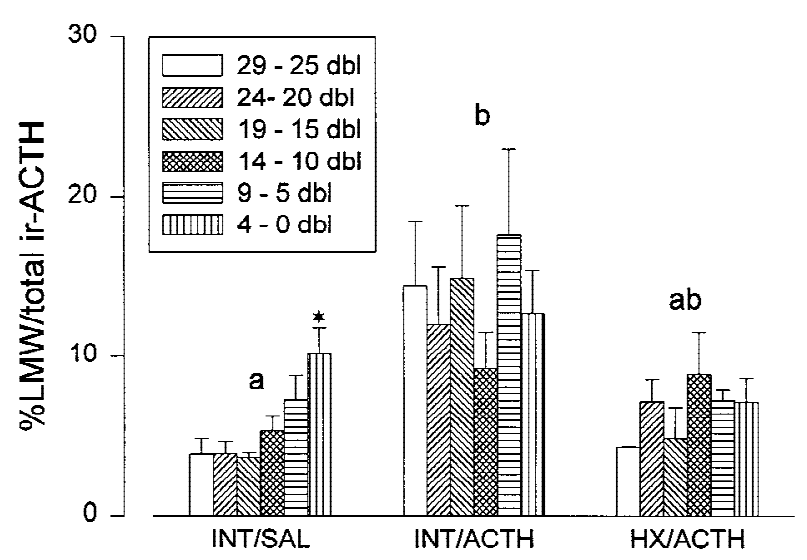

Figure 2 The percentage of LMW ir-ACTH as a proportion of total ir-ACTH in plasma from INT/SAL $(n=4)$, INT/ACTH $(n=7)$ and $\mathrm{HX} / \mathrm{ACTH}(n=3)$ fetuses (only those from ewes that went into labour) from 29-25 days before labour (dbl) to 5-0 days before labour. There was a significant difference in LMW ir-ACTH between the three groups of fetuses. There was a significant increase in the percentage of LMW ir-ACTH with advancing GA in INT/SAL fetuses only. Asterisks indicate bars that are significantly different from others within each treatment group (when compared with the first two GA blocks) and different letters indicate groups that are significantly different from each other as indicated by LSD test. dbl, days before labour.

in INT/ACTH fetuses $3478 \pm 472$ ng.min per $\mathrm{ml}$ and in HX/ACTH fetuses $2423 \pm 277$ ng.min per ml. The mean increase in cortisol concentrations achieved (for all GAs) in INT/SAL fetuses was $8 \cdot 1 \pm 1 \cdot 3 \mathrm{ng} / \mathrm{ml}$, in INT/ACTH fetuses was $43 \cdot 4 \pm 5 \cdot 2 \mathrm{ng} / \mathrm{ml}$, and HX/ACTH fetuses was $29 \cdot 6 \pm 3 \cdot 1 \mathrm{ng} / \mathrm{ml}$. It should be noted that there exists the same rank order for the percentage of LMW ir-ACTH, adrenal responsiveness and the increase in cortisol concentrations, i.e. INT $/ \mathrm{SAL}<\mathrm{HX} / \mathrm{ACTH}<\mathrm{INT} / \mathrm{ACTH}$.

Experiment 3: effect of interrupting the $\mathrm{ACTH}_{(1-24)}$ infusion

The effects on ir-ACTH and cortisol concentrations in INT/ACTH and HX/ACTH fetuses of stopping the maintenance $\mathrm{ACTH}_{(1-24)}$ infusion for $4 \mathrm{~h}$ are presented in

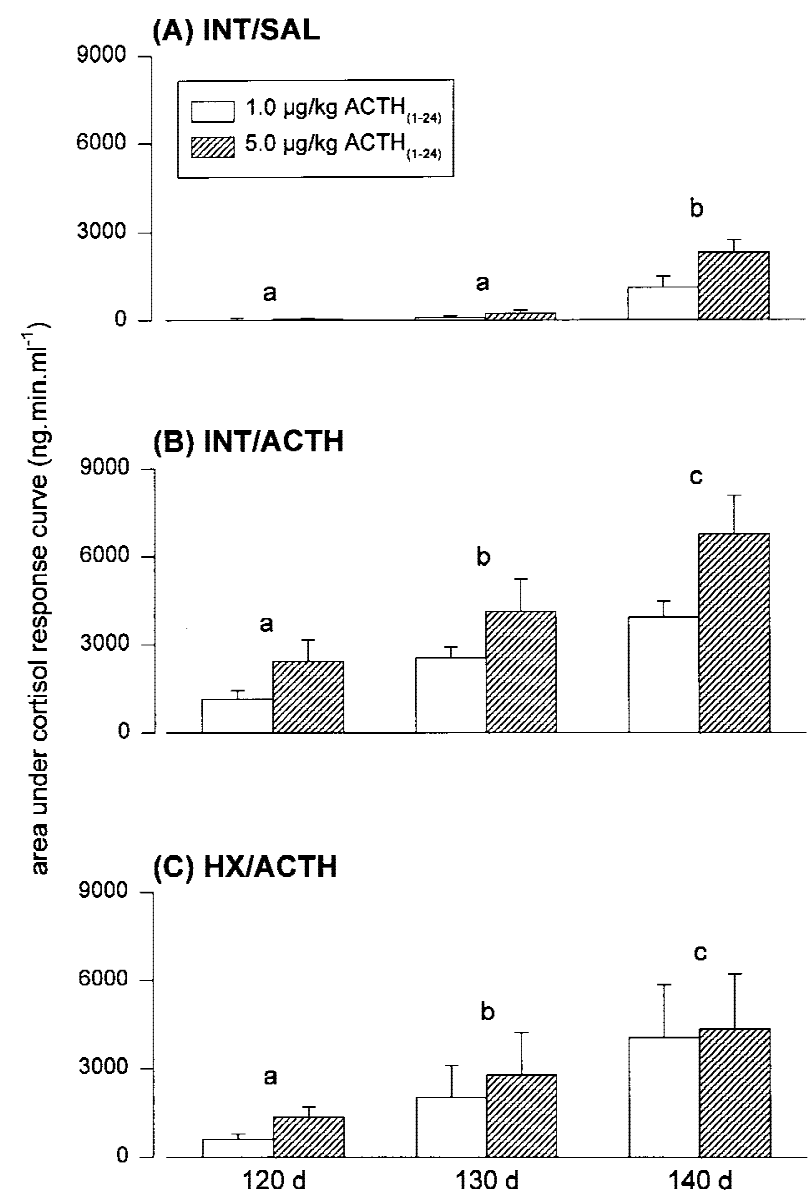

Figure 3 The area under the cortisol response curve (adrenal responsiveness) following the administration of $1.0 \mu \mathrm{g} / \mathrm{kg}$ and $5 \cdot 0 \mu \mathrm{g} / \mathrm{kg} \mathrm{ACTH}_{(1-24)}$ to INT/SAL $(\mathrm{A} ; n=4)$, INT/ACTH (B; $\left.n=4\right)$, and HX/ACTH $(C ; n=6)$ fetuses at 120,130 and 140 days GA. There was a significant difference in adrenal responsiveness between the three groups of fetuses. Adrenal responsiveness in INT/ACTH and HX/ACTH fetuses was significantly greater than that in INT/SAL fetuses; however, there was no significant difference in adrenal responsiveness between INT/ACTH and $\mathrm{HX} / \mathrm{ACTH}$ fetuses. There was a significant increase in adrenal responsiveness with advancing GA in all groups of fetuses. Different letters indicate GAs that were significantly different from each other within each treatment group. 
Table 3 The increases in cortisol concentrations $(\mathrm{ng} / \mathrm{ml})$ achieved following the administration of $1.0 \mu \mathrm{g} / \mathrm{kg}$ and $5.0 \mu \mathrm{g} / \mathrm{kg} \mathrm{ACTH}(1-24)$ at 120,130 and 140 days GA in INT/SAL $(n=4)$, INT/ACTH $(n=4)$ and HX/ACTH $(n=6)$ fetuses (only those from ewes that went into labour). Data are means \pm S.E.M.

\begin{tabular}{|c|c|c|c|c|}
\hline & $\mathbf{A C T H}_{(\mathbf{1 - 2 4})}(\mu \mathrm{g} / \mathrm{kg})$ & INT/SAL $^{x}$ & INT/ACTH ${ }^{y}$ & HXIACTH ${ }^{y}$ \\
\hline \multicolumn{5}{|c|}{ GA (days) } \\
\hline \multirow[t]{2}{*}{120} & $1 \cdot 0$ & $1 \cdot 1 \pm 0 \cdot 5^{\mathrm{a}}$ & $17 \cdot 5 \pm 3 \cdot 7^{\mathrm{a}}$ & $8 \cdot 7 \pm 1 \cdot 3^{\mathrm{a}}$ \\
\hline & $5 \cdot 0$ & $1 \cdot 6 \pm 0 \cdot 7$ & $26 \cdot 7 \pm 6 \cdot 0$ & $13 \cdot 8 \pm 2 \cdot 4$ \\
\hline \multirow[t]{2}{*}{130} & $1 \cdot 0$ & $2 \cdot 8 \pm 0 \cdot 6^{\mathrm{b} *}$ & $43 \cdot 1 \pm 7 \cdot 0^{\mathrm{ab}}$ & $29 \cdot 0 \pm 8 \cdot 5^{\mathrm{ab}}$ \\
\hline & $5 \cdot 0$ & $5 \cdot 1 \pm 1 \cdot 2$ & $48 \cdot 1 \pm 10 \cdot 1$ & $28 \cdot 9 \pm 8 \cdot 9$ \\
\hline \multirow[t]{2}{*}{140} & $1 \cdot 0$ & $20 \cdot 8 \pm 2 \cdot 2^{\mathrm{c} *}$ & $52 \cdot 8 \pm 8 \cdot 1^{b}$ & $52 \cdot 8 \pm 13 \cdot 3^{b}$ \\
\hline & $5 \cdot 0$ & $25 \cdot 3 \pm 2 \cdot 7$ & $72 \cdot 0 \pm 11 \cdot 9$ & $47 \cdot 3 \pm 14 \cdot 2$ \\
\hline
\end{tabular}

Different letters indicate treatment groups $(x, y)$ or GAs (within each treatment group: $a-c)$ that are significantly different from each other as indicated by LSD test. Asterisk indicate GAs at which a signficant difference between the two doses of $\mathrm{ACTH}_{(1-24)}$ was identified by LSD test.

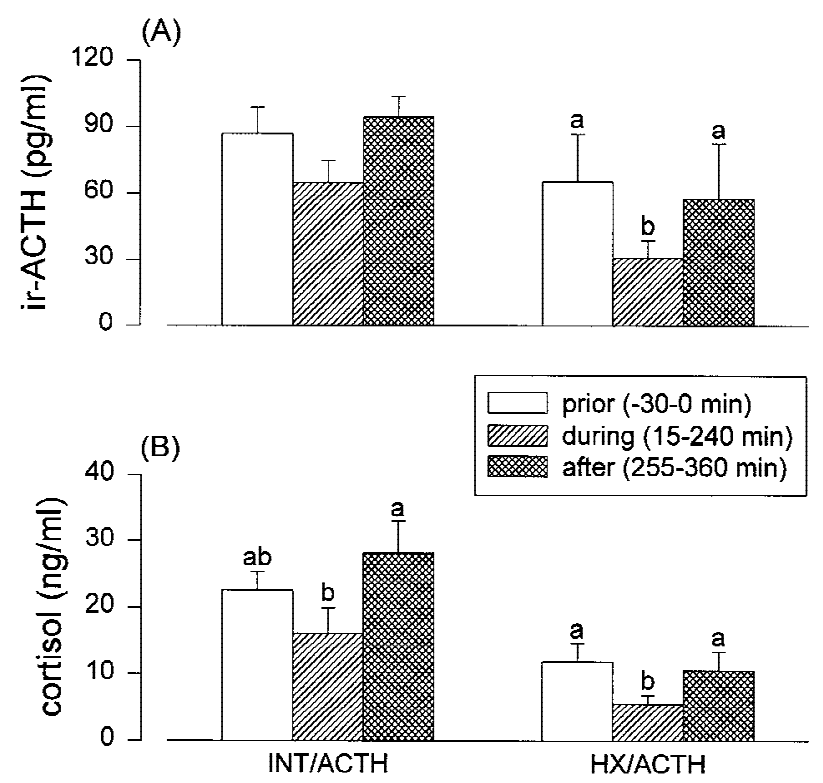

Figure 4 Mean plasma ir-ACTH concentrations ( $\mathrm{A}$; as measured by the Monash RIA) and cortisol concentrations (B) in INT/ACTH $(n=4)$ and $\mathrm{HX} / \mathrm{ACTH}(n=6)$ fetuses prior to $(-30-0 \mathrm{~min})$, during (15-240 $\mathrm{min}$ ) and after (255-360 $\mathrm{min})$ a $4 \mathrm{~h}$ interruption of the maintenance $\mathrm{ACTH}_{(1-24)}$ infusion at $136 \pm 1$ days GA. Different letters indicate time points that are significantly different from each other as indicated by LSD test.

Fig. 4. Data are presented as means of the samples taken prior to ( -30 to $0 \mathrm{~min})$, during $(15-240 \mathrm{~min})$ and after (255-360 min) the interruption of the $\mathrm{ACTH}_{(1-24)}$ infusion. In INT/ACTH fetuses, there was no significant difference in ir-ACTH concentrations as a result of interrupting the $\mathrm{ACTH}_{(1-24)}$ infusion. Cortisol concentrations in INT/ACTH fetuses were significantly lower during the interruption of the infusion than after its recommencement, though the values obtained prior to the interruption were not significantly different to any other time period. In $\mathrm{HX} / \mathrm{ACTH}$ fetuses, the interruption of the $\mathrm{ACTH}_{(124)}$ infusion led to a significant decrease in both ir-ACTH and cortisol concentrations. The recommencement of the $\mathrm{ACTH}_{(1-24)}$ infusion restored the concentrations of both hormones in these fetuses.

\section{Discussion}

The central finding of this study is that term parturition can occur in both intact and HX fetuses provided with a constant, maintenance infusion of $\mathrm{ACTH}_{(1-24)}$. This result confirms and extends the finding of Jacobs et al. (1994) that this infusion of $\mathrm{ACTH}_{(1-24)}$ is sufficient to permit the maturation of the fetal adrenal cortex and the onset of labour at the expected time in HX fetuses. In addition, we have demonstrated that the same $\mathrm{ACTH}_{(1-24)}$ infusion regimen in intact fetuses is not sufficient to significantly advance parturition or adrenal maturation. Labour in all groups of fetuses in this study was characterised by an increase in plasma cortisol concentrations beginning around 14 days before term, as shown previously (Bassett \& Thorburn 1969, Jacobs et al. 1994), but this was not associated with an increase in plasma ir-ACTH concentrations in $\mathrm{ACTH}_{(1-24)}$-infused HX fetuses. This observation was confirmed by assaying ir-ACTH concentrations in two distinct RIAs, thus reinforcing the biological conclusions which may be drawn from the data. In contrast to HX/ACTH fetuses, increases in ir-ACTH concentrations were observed using both RIAs in both saline- and $\mathrm{ACTH}_{(1-24)}$-infused intact fetuses. Thus, a late gestation increase in fetal ir-ACTH concentrations appears not to be an absolute prerequisite for parturition in the sheep.

Adrenal cortical growth, as assessed morphometrically, and adrenal maturation, reflected in basal cortisol concentrations, were similar in all groups of fetuses. It has previously been suggested that HMW ACTH-containing peptides antagonise the steroidogenic effect of LMW ACTH on the fetal adrenal and that the ratio of HMW to LMW ACTH-containing peptides is an important 
determinant of basal cortisol secretion and adrenal growth (Roebuck et al. 1980, Thorburn \& Liggins 1994). In plasma from INT/ACTH fetuses, the proportion of LMW ir-ACTH was significantly greater than that found in INT/SAL fetuses. This increase in LMW ir-ACTH was not associated with any acceleration of adrenal maturation or the timing of parturition, as demonstrated by adrenal size and basal cortisol concentrations. Furthermore, as basal cortisol concentrations and adrenal size did not differ between INT/SAL and INT/ACTH fetuses, it is unlikely that the $\mathrm{ACTH}_{(1-24)}$ infusion rate exceeded the normal regulatory capabilities of the fetal hypothalamopituitary-adrenal axis. We therefore suggest that the ratio of LMW to HMW ACTH is not the singular determinant of basal cortisol secretion. The finding that HX/ACTH fetuses underwent normal adrenal growth and maturation indicates that pituitary-derived fragments of pro-opiomelanocortin (POMC) (N-terminal $\mathrm{POMC}_{(1-77)}$ and its congeners) may not be necessary for mitogenesis in the adrenal cortex (Estivariz et al. 1982, Saphier et al. 1993) since $\mathrm{ACTH}_{(1-24)}$ alone appears to be sufficient to restore normal adrenal growth and function in HX fetuses.

In order to determine the contribution of the $\mathrm{ACTH}_{(1-24)}$ infusion to measured ir-ACTH concentrations, the infusion was interrupted for a $4 \mathrm{~h}$ period in HX/ACTH and INT/ACTH fetuses. The reduction in ir-ACTH concentrations following the cessation of the $\mathrm{ACTH}_{(1-24)}$ infusion in HX/ACTH fetuses, and the consequent fall in plasma cortisol concentrations, demonstrates the absolute requirement in the $\mathrm{HX}$ fetus for exogenous ACTH to maintain basal cortisol concentrations. In these fetuses, ir-ACTH concentration did not fall to zero when the $\mathrm{ACTH}_{(1-24)}$ infusion was turned off, consistent with previous reports of extra-pituitary sources of ACTH-immunoreactive material in the fetus (Challis \& Brooks 1989, Cudd \& Wood 1995). In contrast, in INT/ACTH fetuses, there were more modest changes in ir-ACTH and cortisol concentrations following the cessation of the $\mathrm{ACTH}_{(1-24)}$ infusion. We interpret these changes as reflecting the ability of the fetus with an intact HPA axis to respond to the withdrawal of the $\mathrm{ACTH}_{(1-24)}$ infusion with an appropriate increase in endogenous ACTH secretion. The increase in the cortisol concentrations following the recommencement of the infusion most probably represents an acute response to the return of the exogenous stimulus to cortisol release. In the long term, however, as reflected by the basal cortisol concentrations over the entire study period, the INT/ACTH fetus appears to be able to titrate its endogenous ACTH secretion to ensure physiologically appropriate concentrations of circulating cortisol. We believe that this argument is supported by the observation that plasma ir-ACTH concentrations over the study period do not differ between the INT/SAL and INT/ACTH fetuses, which may be consistent with some reduction in endogenous ACTH secretion during the $\mathrm{ACTH}_{(1-24)}$ infusion.

In the previous study of Jacobs et al. (1994), fetal HX was performed at 125 days GA, while in the present study, this procedure was carried out at $115 \pm 1$ days GA. It is important to note that the GA at which HX took place did not affect the timing of parturition and that the initiation of labour was not simply related to the length of time fetuses were exposed to the $\mathrm{ACTH}_{(1-24)}$ infusion. It therefore seems unlikely that the $\mathrm{ACTH}_{(1-24)}$ infusion had a cumulative effect upon the fetal adrenal gland, such that a given mass or time of exposure to $\mathrm{ACTH}_{(1-24)}$ is sufficient to explain the onset of parturition. It was also reported in the study of Jacobs et al. (1994) that, in contrast to the present study, ir-ACTH concentrations in HX/ ACTH fetuses were undetectable. It has now been found that, in contrast to what was previously stated, the immunoradiometric assay used in the former study to measure plasma ir-ACTH concentrations was not able to detect $\mathrm{ACTH}_{(1-24)}$, the peptide infused into HX fetuses. The assays used in the present study measure $\mathrm{ACTH}_{(1-24)}$, $\mathrm{ACTH}_{(1-39)}$ and the HMW ACTH precursors and therefore permit a more reliable assessment of the ir-ACTH concentrations achieved in HX/ACTH fetuses.

In both the present study and that of Jacobs et al. (1994) not all ewes with HX fetuses receiving an $\mathrm{ACTH}_{(1-24)}$ infusion proceeded to labour by 161 days GA. The one HX/ACTH fetus of the present study which did not deliver by 161 days GA was found to have small adrenal glands at the time of elective post mortem, suggesting that the $\mathrm{ACTH}_{(1-24)}$ infusion was below the necessary threshold to maintain adrenal growth in this animal. We have no coherent explanation of why the basal fetal cortisol concentrations appeared to rise normally in this animal and then fell precipitously without the successful initiation of labour, except to suggest that there may have been a late, but unrecognised, fault in the administration of the infusion. It must be stated, however, that we have no evidence to support this idea, and we believe that it is not surprising, from a pharmacological perspective, that individual variation between animals leads to individual variation in the outcome of treatments.

A further important aspect of this study is that maintenance $\mathrm{ACTH}_{(1-24)}$ infusion was shown to prematurely increase adrenal responsiveness in the late-gestation ovine fetus. Since there was a correlation, in rank order, between the extent of the increase in adrenal responsiveness and that of plasma LMW ir-ACTH levels, we suggest that the ratio of LMW to HMW ACTH may be a determinant of fetal adrenal responsiveness. This ratio, however, appears not to be the only regulator of adrenal responsiveness, since adrenal responsiveness increased from 120 days to 140 days GA in all groups of fetuses, over which time discernible increases in LMW ir-ACTH levels were only observed in INT/SAL fetuses. The present data are consistent with previous in vitro studies that have shown 
that the HMW ACTH-containing peptides inhibit the ability of fetal adrenal cells to respond to ACTH (Roebuck et al. 1980, Schwartz et al. 1995).

In this study we have demonstrated a dissociation between the emergence of adrenal responsiveness and both basal cortisol secretion and the timing of parturition. In all $\mathrm{ACTH}_{(1-24)}$-infused fetuses, the level of adrenal responsiveness, even at the earliest GA measured (120 days), was similar in magnitude to that achieved in control fetuses only by 140 days GA. Despite this markedly enhanced ability to respond to $\mathrm{ACTH}_{(1-24)}$, these fetuses failed to deliver prematurely. Since basal cortisol concentrations were not affected by changes in the ratio of LMW to HMW ACTH, we suggest that there is a differential regulation of basal cortisol output and the ability of the fetal adrenal gland to respond to ACTH stimulation. This is an important finding since it has been proposed that enhanced adrenal responsiveness to ACTH regulates the pre-partum cortisol surge (Challis \& Brooks 1989, Thorburn \& Liggins 1994).

This study found that fetal body weights were smaller in $\mathrm{ACTH}_{(1-24)}$-infused fetuses, though the cause and significance of this observation is difficult to discern. While cortisol has well-recognised catabolic effects in the ovine fetus (Barnes et al. 1977), we were unable to detect, measuring total cortisol concentrations every 1 or 2 days, any differences between the three groups of fetuses. It remains formally possible that free cortisol concentrations or the circadian rhythms of cortisol may differ between the different groups of fetuses, but we have no evidence to support either of these hypotheses. Furthermore, and perhaps more importantly, we have been unable to repeat the observation of decreased fetal body weight in subsequent studies involving $\mathrm{ACTH}_{(1-24)}$-infused intact fetuses (data not shown) and Jacobs et al. (1994) found no such effect in $\mathrm{ACTH}_{(1-24)}$-infused HX fetuses. We did consider, however, whether the differences in fetal body weight observed in the present study might contribute to altered ACTH kinetics during the adrenal responsiveness testing, and, if this were to occur, whether it may partly explain the differences in adrenal responsiveness observed between the three groups of fetuses. When we measured maximum ir-ACTH concentrations achieved after $\mathrm{ACTH}_{(1-24)}$ administration, or the apparent half-life of $\mathrm{ACTH}_{(1-24)}$ following these tests, we failed to identify any differences between the different groups of fetuses (data not shown). Accordingly, we consider the apparently decreased body weight in the $\mathrm{ACTH}_{(1-24)}$-infused fetuses a phenomenon of limited physiological significance.

In conclusion, these results demonstrate that, while the presence of $\mathrm{ACTH}$ is required to prevent adrenal atrophy and allow adrenocortical maturation, an increase in ACTH concentration in late gestation does not appear to be an absolute prerequisite for parturition. Indeed, a constant plasma ACTH concentration appears sufficient for adrenal maturation and term parturition in the absence of a pituitary. Furthermore, no relationship was found between the ratio of LMW to HMW ACTH species and basal cortisol secretion, or the timing of labour. This ratio may, however, regulate the ability of the fetal adrenal gland to respond to $\mathrm{ACTH}$, since adrenal responsiveness was elevated in those fetuses that had elevated LMW ir-ACTH levels. The increase in adrenal responsiveness in ACTH $_{(1-24)}$-infused fetuses was not associated with a change in basal cortisol concentrations or the timing of parturition. These findings therefore bring into question the precise role of ACTH in the adrenal development seen in the late gestation ovine fetus and leave unanswered the relationship between heightened adrenal responsiveness and basal cortisol concentrations and the timing of parturition.

\section{Acknowledgements}

We are grateful to A Satragno for surgical assistance, D Caddy for statistical advice and M Serapiglia and J Loose for valuable technical assistance. The authors would also like to thank G Rice for the donation of the ACTH antiserum and G Jenkin for his assistance with the preparation of this manuscript. This study was supported by a grant from the NH\&MRC to I R Y, B J C and G D T.

\section{Dedication}

This manuscript is dedicated to the memory of Professor Geoffrey D Thorburn for his invaluable contribution to these experiments and his enthusiasm for the study of fetal physiology.

\section{References}

Antolovich GC, McMillen IC, Robinson PM, Silver M, Young IR \& Perry RA 1991 The effect of hypothalamo-pituitary disconnection on the functional and morphologic development of the pituitaryadrenal axis in the fetal sheep in the last third of gestation. Neuroendocrinology $\mathbf{5 4}$ 254-261.

Barnes RJ, Comline RS \& Silver M 1977 The effects of bilateral adrenalectomy or hypophysectomy of the foetal lamb in utero. Journal of Physiology 264 429-447.

Bassett JM \& Thorburn GD 1969 Foetal plasma corticosteroids and the initiation of parturition in sheep. Journal of Endocrinology 44 285-286.

Bocking AD, McMillen IC, Harding R \& Thorburn GD 1986 Effect of reduced uterine blood flow on fetal and maternal cortisol. Journal of Developmental Physiology 8 237-245.

Carr GA, Jacobs RA, Young IR, Schwartz J, White A, Crosby S \& Thorburn GD 1995 Development of adrenocorticotropin-(1-39) and precursor peptide secretory responses in the fetal sheep during the last third of gestation. Endocrinology 136 5020-5027.

Challis JRG \& Brooks AN 1989 Maturation and activation of hypothalamic-pituitary-adrenal function in fetal sheep. Endocrine Reviews 10 182-204 
Cloete JHL 1939 Prenatal growth in the Merino sheep. Onderstepoort Journal of Veterinary Science and Animal Industry 13 417-558.

Cudd TA \& Wood CE 1995 Secretion and clearance of immunoreactive ACTH by fetal lung. American Journal of Physiology 268 E845-E848.

Deayton JM, Young IR, Hollingworth SA, White A, Crosby SR \& Thorburn GD 1994 Effect of late hypothalamo-pituitary disconnection on the development of the HPA axis in the ovine fetus and the initiation of parturition. Journal of Neuroendocrinology 6 25-31.

Durand P, Cathiard A-M \& Saez JM 1982 In vitro maturation of ovine fetal adrenal cells adenylate cyclase: corticotropin-dependent and independent development of the response to corticotropin. Biochemical and Biophysical Research Communications 106 8-15.

Durand P, Cathiard AM \& Saez JM 1985 Maturation of the ovine fetal adrenal gland: in vivo and in vitro studies. In The Endocrine Physiology of Pregnancy and the Peripartal Period. Serono Symposia Publications vol 21, pp 31-52. Eds RB Jaffe \& S Dell'Acqua. New York: Raven Press.

Estivariz FE, Iturriza F, McLean C, Hope J \& Lowry PJ 1982 Stimulation of adrenal mitogenesis by N-terminal proopiocortin peptides. Nature 297 419-422.

Glickman JA \& Challis JRG 1980 The changing response pattern of sheep fetal adrenal cells throughout the course of gestation. Endocrinology 106 1371-1376.

Hollingworth SA, Deayton JM, Young IR \& Thorburn GD 1995 Prostaglandin $\mathrm{E}_{2}$ administered to fetal sheep increases the plasma concentrations of adrenocorticotropin (ACTH) and the proportion of ACTH in low molecular weight forms. Endocrinology 136 1233-1240.

Jacobs RA, Young IR, Hollingworth SA \& Thorburn GD 1994 Chronic administration of low doses of adrenocorticotropin to hypophysectomized fetal sheep leads to normal term labor. Endocrinology 134 1389-1394.

Jones CT \& Roebuck MM 1980 ACTH peptides and the development of the fetal adrenal. Journal of Steroid Biochemistry 12 77-82.

Jones CT, Boddy K, Robinson JS \& Ratcliffe JG 1977 Developmental changes in the responses of the adrenal glands of foetal sheep to endogenous adrenocorticotrophin, as indicated by hormone responses to hypoxaemia. Journal of Endocrinology 72 279-292.

Jones CT, Edwards AV \& Tindell D 1992 Inhibitory effects of proopiomelanocortin on cortical and medullary activity in the calf adrenal. Journal of Developmental Physiology 17 69-73.
Liggins GC 1968 Premature parturition after infusion of corticotrophin or cortisol into foetal lambs. Journal of Endocrinology 42 323-329.

Liggins GC, Kennedy PC \& Holm LW 1967 Failure of initiation of parturition after electrocoagulation of the pituitary of the fetal lamb. American Journal of Obstetrics and Gynecology 98 1080-1086.

Mesiano S, Young IR, Baxter RC, Hintz RL, Browne CA \& Thorburn GD 1987 Effect of hypophysectomy with and without thyroxine replacement on growth and circulating concentrations of IGF-I and II in the fetal lamb. Endocrinology 120 1821-1830.

Ozolins IZ, Young IR \& McMillen IC 1992 Surgical disconnection of the hypothalamus from the fetal pituitary abolishes the corticotrophic response to intrauterine hypoglycemia or hypoxemia in the sheep during late gestation. Endocrinology 130 2438-2445.

Roebuck MM, Jones CT, Holland D \& Silman R 1980 In vitro effects of high molecular weight forms of ACTH on the fetal sheep. Nature 284 616-618.

Rose JC, Meis PJ, Urban RB \& Greiss FC 1982 In vitro evidence for increased adrenal sensitivity to adrenocorticotropin-(1-24) in the lamb fetus late in gestation. Endocrinology 111 80-85.

Saphier PW, Glynn BP, Woods RJ, Sheperd DAL, Jeacock K \& Lowry PJ 1993 Elevated levels of N-terminal pro-opiomelanocortin peptides in fetal sheep plasma may contribute to fetal adrenal gland development and the pre-parturient cortisol surge. Endocrinology 133 1459-1461.

Schwartz J, Kleftogiannis F, Jacobs R, Thorburn GD, Crosby SR \& White A 1995 Biological activity of adrenocorticotropic hormone precursors on ovine adrenal cells. American Journal of Physiology 268 E623-E629.

Thorburn GD \& Liggins GC 1994 Role of the fetal pituitary-adrenal axis and placenta in the initiation of parturition. In Marshall's Physiology of Reproduction, vol 3, pp 1003-1036. Ed. GE Lamming. London: Chapman and Hall.

Wintour EM, Brown EH, Denton DA, Hardy KJ, McDougall JG, Oddie CJ \& Whipp GT 1975 The ontogeny and regulation of corticosteroid secretion by the ovine foetal adrenal. Acta Endocrinologica 79 301-316.

Received 15 September 1997

Revised manuscript received 16 January 1998 Accepted 9 March 1998 